\title{
The Importance of Galectin 3 for Risk Stratification and Prognosis in Hypertrophic Cardiomyopathy
}

\author{
Hakan UÇAR ${ }^{1}$, Sinem ÖZYILMAZ ${ }^{1}$, Muhammet Hulusi SATILMIŞOĞLU², \\ Hamdi PUSUROĞLU ${ }^{2}$
}

\begin{abstract}
Objective: Galectin-3 levels were found to be associated with worse prognosis in various cardiac disorders. The aim of the study was to assess the relationship between serum galectin-3 levels and prognosis among HCM patients.

Material and Methods: This study included 107 consecutive patients with HCM. Galectin-3 levels were evaluated in all participants and the HCM Risk-SCD calculated in patients with HCM for each patient.
\end{abstract}

Results: The low galectin-3 and high galectin-3 groups showed significant differences in NYHA class, the HCM Risk SCD, the HCM Risk SCD (>6\%), interventricular septum thickness, left ventricular mass(LVM), and LVM index, and percentage of ventricular extra systole, ventricular tachycardia, cardiopulmonary resuscitation, ICD implantation, shock, admitted hospital with heart failure symptoms into two groups(all $\mathrm{p}<0.05)$. The low galectin-3 HCM patients $(\mathrm{n}=62)$ had galectin-3 levels of $4.720 \pm 2.202 \mathrm{ng} / \mathrm{ml}$ and the high galectin-3 HCM patients $(\mathrm{n}=45)$ had galectin-3 levels of $7.943 \pm 1.618 \mathrm{ng} / \mathrm{ml}(\mathrm{p}<0.001)$. Both in the univariate and multivariate analysis, galectin-3 determined that the HCM Risk SCD is an independent predictor of high-risk $(\mathrm{p}<0.001)$. In ROC curve analysis, a galectin-3 $>6.324 \mathrm{ng} / \mathrm{ml}$ was identified as an effective cut-off point in the HCM Risk SCD for HCM (area under curve $=0.878,95 \% \mathrm{CI}=0.815-0.942, \mathrm{p}$ $<0.001$ ). A galectin-3 value of more than $6.324 \mathrm{ng} / \mathrm{ml}$ yielded a with sensitivity, specificity, positive predictive value and negative predictive value of, $83 \%, 82 \%$, $73 \%, 93 \%$.

Conclusion: These results shape the concept of considering galectin-3 as a new target for therapeutic intervention or recognizing patients with high risk for SCD

1 Department of cardiology, Faculty of Medicine, Istanbul Aydin University, VM Florya Medical Park Hospital, Istanbul, Turkey

2 Department of cardiology, Mehmet Akif Ersoy Thoracic and Cardiovascular Surgery Training and Research Hospital, Istanbul, Turkey

DOI: 10.17932/EJOH.2020.022/ejoh_v01i1002 
or malign arrythmias. In this study, galectin-3 is an independent predictor of high risk for HCM Risk-SCD in HCM. Therefore, an early recognition of high-risk patients for SCD and intervention with new anti-inflammatory and antifibrotic agents might provide additional benefit over existing treatment strategies.

Keywords: Galectin 3, sudden cardiac death, risk stratification, prognosis, hypertrophic cardiomyopathy

\section{Introduction}

Sudden cardiac death (SCD) is well recognized and most frightening complication of hypertrophic cardiomyopathy (HCM). Most cases remain asymptomatic or rarely symptomatic (1) in their lifetime or sometimes SCD may be the first symptom. ICD therapy is an important treatment option for preventing SCD and improving prognosis. However, it can cause some unwanted complications like infective endocarditis or inappropriate ICD shocks (2). For these reasons, it is important to choose the patient who really needs ICD treatment. Several invasive and noninvasive studies have been done for risk stratification of SCD since the disease was first defined (3). One of the reasons for vigorous efforts among this subject is identification of patients who are at high risk for ventricular arrhythmias or SCD and to make the right choice when selecting patients for ICD implantation. In order to solve this problem ESC guideline recommends the use of a new risk of SCD calculation model According to this guideline implantable cardioverter defibrillator (ICD) implantation should be considered in patients with high risk $(1,4)$.

Galectin-3 is a beta-galactoside-binding lectin that is expressed by macrophages. Nowadays, studies have shown that Galectin-3 mediate cardiac fibrosis or inflammation particularly in patients with heart failure. Moreover galectin-3 have all been well validated to predict death and heart failure (5) following a MI and provide risk stratification information for heart failure. In this study, we aimed to investigate the relationship between serum Galectin-3 levels and HCM RiskSCD.

\section{Material and Methods}

Patient Population

This prospective observational study included 107 consecutive patients with HCM and a control group of 80 subjects who presented to the Mehmet Akif Ersoy Thoracic and Cardiovascular Surgery Center, Training and Research 
Hospital between December 2012 and March 2016. The study was approved by the ethics committee. All patients and healthy subjects signed informed consent forms. Only adult patients ( $>17$ years) were included into the study. Patients whose echocardiography (ECHO) revealed HCM or patients with positive gene mutations or patients with HCM-specific late gadolinium enhancement or the presence of apical hypertrophy assessed by cardiac magnetic resonance (MR) imaging were included in the study. Hypertrophic cardiomyopathy defined as a maximum LV wall thickness $\geq 15 \mathrm{~mm}$ in one or more LV myocardial segments as measured with any imaging technique with unexplained abnormal loading conditions patients with lesser degrees of wall thickening (13-14 mm); patients with a family history, and electrocardiogram (ECG) abnormalities (1).

\section{Exclusion criteria}

We excluded from our analysis the patients who do not want to participate in the study. Patients with a prior history of the ICD or permanent pacemaker implantation for secondary protection or prior cardiac arrest and sustained ventricular tachycardia; patients with prolonged hypertension, renal failure, previous coronary artery disease, moderate to severe valve diseases, atrial fibrillation, metabolic storage disease, an ejection fraction less than $50 \%$, wall motion abnormalities. Patients with a body mass index (BMI) over $30 \mathrm{~kg} / \mathrm{m} 2$ were excluded from the study. In this study the maximum LV wall thickness was $33 \mathrm{~mm}$ patients whose LV wall thickness was $\geq 35 \mathrm{~mm}$ was not included into the study. The final study population consisted of 107 patients.

\section{Electrocardiography}

A 12-derivation surface ECG was obtained with using a Nihon Kohden-Cardiofax $\mathrm{S}$ instrument (ECG-1250K, filter range $0.5 \mathrm{~Hz}$ to $150 \mathrm{~Hz}, \mathrm{AC}$ filter $60 \mathrm{~Hz}$, at a speed of $25 \mathrm{~mm} / \mathrm{s}$ and an amplitude of $10 \mathrm{~mm} / \mathrm{mV}$; Nihon Kohden, Tokyo, Japan) in all patients in supine position. The rhythm, speed, and QRS morphology were assessed.

\section{Measurement of plasma galectin-3 levels}

Serum galectin-3 was measured, in duplicate, using a commercially available enzyme-linked immunosorbent assay method (Human galectin-3 ELISA kit, catalogue no. DGAL30; R\&D Systems, Inc., Minneapolis, Minnesota, USA). The intraobserver variability in the measurements of galectin-3 was also assessed and all of the mean intra-assay coefficients of variance were less than $4.6 \%$. 


\section{Echocardiography}

Upon hospital admission, a transthoracic echocardiographic study was performed using a Vivid S5 system (General Electric Vivid S5; GE Vingmend Ultrasound AS, Horten, Norway) with a 1.7/3.4 MHz phased-array transducer. All echocardiographic parameters were measured off-line, and an average of three cardiac cycles was used. Biplane Simpson method was used to calculate LV ejection fraction (LVEF) (6). LV wall thickness (interventricular septum, posterior wall) left ventricular end diastolic diameter (LVEDD), and LV end systolic diameter (LVESD) were measured in the parasternal long axis. The LV outflow tract obstruction gradient (LVOTOG) was measured in the apical five chambers while the patients were performing the Valsalva maneuver. In addition, the LV end diastolic volume, end systolic volume, left atrial diameter (LAD), left atrial volume (LAV), left atrial volume index (LAVI), and LV mass in grams were calculated from M-mode echocardiograms according to the formulas described by Devereux et al. (7). The LV mass was indexed to body surface area as the LV mass index (LVMI) in $\mathrm{g} / \mathrm{m}^{2}$ of body surface area. Mitral valve regurgitation (systolic anterior motion of the mitral valve) and the relative wall thickness index (RWTI) for LV diastolic dysfunction were also evaluated.

\section{Holter electrocardiography}

The analyses were made from the 12 channel recordings obtained from the ambulatory holter monitors (DMS 300-7 Holter Reader; DSM, Stateline, NV, USA) for a period of 24 hours in all patients. Before automatic analysis, the tapes were analyzed using the Holter program (CardioScan 12.0 DM software, DSM). The recordings were evaluated for rhythm, supraventricular extrasystole (SVES), supraventricular tachycardia (SVT), paroxysmal atrial fibrillation (PAF), ventricular extrasystole (VES), non-sustained and/or sustained ventricular tachycardia (NSVT), and atrioventricular (AV) block with pauses

Measurement of the predicted five-year risk of SCD for the patients

The HCM Risk SCD risk assessment was performed to each patient with HCM risk of SCD calculator on the first visit and it was repeated if there were any change in patient's clinical status. The patients with a predicted HCM risk of SCD were divided into two groups based on percentage, as follows: $\leq 5.9 \%$ group, and the $>6 \%$ group $(1,4)$.

Study endpoints and follow-up

On admission, all demographic characteristics of patients were recorded. Patients 
were regularly followed during visits to $\mathrm{HCM}$ outpatient clinic at regular 3-month intervals. Any change in clinical status was noted. ECG and ambulatory 24-hour ECG monitoring were performed every 3 months. The primary endpoint for the study was ventricular arrhythmic event. The secondary endpoint was occurrence of major arrhythmic event. Follow-up for clinical endpoints was performed by telephone interview and review of outpatient and inpatient medical records.

Statistical analysis

The study population was divided into 2 groups based on the galectin-3 levels: the first group (galectin-3 high $>6.324 \mathrm{ng} / \mathrm{ml}, \mathrm{n}=45$ ) and the second group (galectin-3 low $(\leq 6.324 \mathrm{ng} / \mathrm{ml}, \mathrm{n}=62)$. Using ROC analysis, Galectin-3 cut off value was determined as $6.324 \mathrm{ng} / \mathrm{ml}$ for HCM-Risk SCD. Continuous variables were expressed as mean \pm standard deviation; categorical variables were defined as percentages. The normally distributed continuous variables were assessed using the Kolmogorov-Smirnov test. A comparison of the parametric values of the 2 groups was performed using a two-tailed Student's $t$ test and for parametric values using Mann Whitney U Test. The categorical variables were compared using the odds ratio, Chi-square test, or Fisher's exact test. Pearson correlation coefficient was used to compare the parametric values of the two groups, and the Spearman correlation coefficient was used for nonparametric comparisons. A backward stepwise logistic regression analysis, which included variables with $\mathrm{P}<0.1$, was performed to identify independent predictors of HCM-Risk SCD. The accuracy of relevant variables from the regression analysis to differentiate between galectin-3 high and galectin-3 low was assessed with receiver operating characteristics (ROC) curves to determined area under the curve and optimal sensitivity and specificity. The statistical evaluation of the data was performed using the SPSS for Windows, Version 22.0 software package (SPSS Inc., Chicago, IL, USA). A P value of less than 0.05 was considered statistically significant.

\section{Results}

Baseline characteristics

A total of 107 hypertrophic cardiomyopathy patients were included in the study. Patients were divided into two groups as patients with a galectin- $3>6.324 \mathrm{ng} / \mathrm{ml}$ and patients with galectin- $3 \leq 6.324 \mathrm{ng} / \mathrm{ml}$. Baseline characteristics, which is shown in Table 1 (average age respectively: 48.7 15.4, $43.2 \pm 14.1$ years; 43 females, 64 males). Statistically significant differences were determined between 2 groups in NYHA (\%) class, plasma Galectin-3 levels, LAV (ml), LAVI (ml/m2), IVST $(\mathrm{mm}), \operatorname{LVM}(\mathrm{g}), \operatorname{LVMI}(\mathrm{g} / \mathrm{m} 2)$, ventricular tachycardia (\%), cardiopulmonary resuscitation (\%), ICD implantation (\%), appropriate shock (\%), admitted with 
heart failure (\%) (all $\mathrm{p}<0.05)$. The low Galectin-3 HCM patients $(\mathrm{n}=62)$ had galectin-3 levels of $4.035 \pm 1.464 \mathrm{ng} / \mathrm{ml}$ and the high Galectin-3 HCM patients (n $=45)$ had galectin-3 levels of $8.226 \pm 1.412 \mathrm{ng} / \mathrm{ml}(\mathrm{p}<0.001)$ (Figure 1).

Clinical outcomes

Mean follow up time is $31.1 \pm 13.2$ months and low Galectin-3 group 27.1 \pm 13.4 months, the high Galectin-3 group 35.9 \pm 11.3 months. During the fallow up time 1 patient was died and 33 patients were hospitalized due to a worsening of heart failure symptoms. Thirty-six patients presented with pre-syncope and 12 patients presented with syncope. A total of 100 of the patients used beta blockers, 4 used amiodarone, 8 used disopyramide and 4 used calcium channel blockers. ICD implanted in 12 patients 8 patients presented with appropriate ICD shock, 3 patients presented with inappropriate ICD shock. Twenty-four patients had nun sustained ventricular tachycardia attack. Twelve patients received CPR (Table 1).

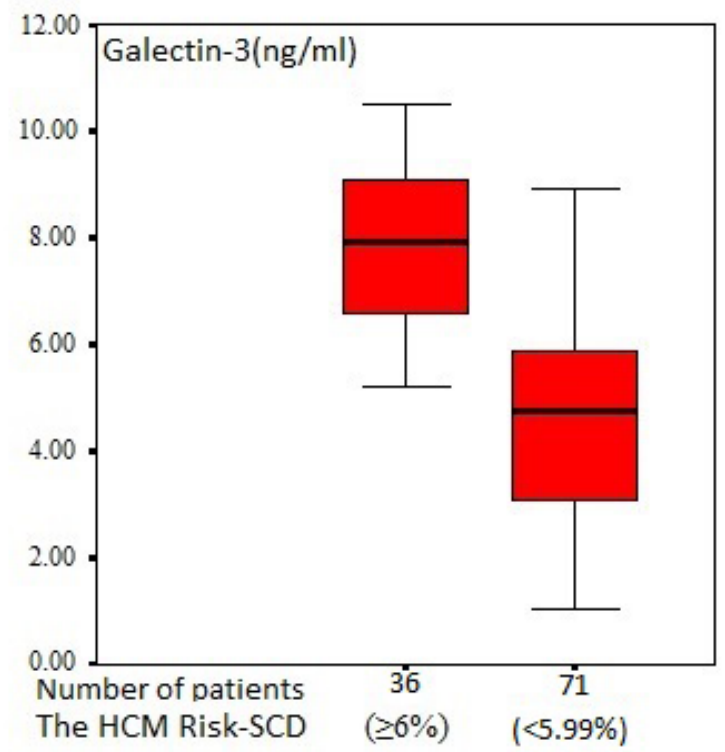

Figure 1. Galectin 3 levels according to HCM Risk $S C D \geq 6 \%$ and $<5.99 \%$ groups. 
Hakan UÇAR, Sinem ÖZYILMAZ, Muhammet Hulusi SATILMIŞOĞLU, Hamdi PUSUROĞLU

Table 1. Baseline and clinical characteristics of all patients

\begin{tabular}{|c|c|c|c|c|c|}
\hline Variabilities & & All(n:107) & $\begin{array}{l}\text { Low } \\
\text { Galectin- } \\
3(\leq 6.324 \mathrm{ng} / \mathrm{m} \\
\text { l) (n:62) }\end{array}$ & $\begin{array}{l}\text { High } \\
\text { Galectin- } \\
\text { 3(>6.324ng/m } \\
\text { l) (n:45) }\end{array}$ & $\begin{array}{l}P \\
\text { value }\end{array}$ \\
\hline Age(years) & & $46.6 \pm 15.1$ & $48.7 \pm 15.4$ & $43.2 \pm 14.1$ & 0.065 \\
\hline \multirow{2}{*}{ Gender } & Male(\%) & $64(60)$ & $37(58)$ & $27(42)$ & \multirow{2}{*}{1.000} \\
\hline & Female $(\%)$ & $43(40)$ & $25(58)$ & $18(42)$ & \\
\hline \multicolumn{2}{|c|}{ Follow up(month) } & $31.1 \pm 13.2$ & $27.1 \pm 13.4$ & $35.9 \pm 11.3$ & 0.001 \\
\hline \multicolumn{2}{|l|}{ BMI $\left(\mathrm{kg} / \mathrm{m}^{2}\right)$} & $26.7 \pm 3.5$ & & $26.5 \pm 3.4$ & 0.138 \\
\hline \multirow{2}{*}{$\begin{array}{l}\text { Diabetes } \\
\text { mellitus(\%) }\end{array}$} & $(+)$ & 5 & $2(40)$ & $3(60)$ & \multirow{2}{*}{0.648} \\
\hline & $(-)$ & 102 & $60(59)$ & $42(41)$ & \\
\hline \multirow{2}{*}{ Hypertension(\%) } & $(+)$ & 4 & $1(25)$ & $3(75)$ & \multirow{2}{*}{0.309} \\
\hline & $(-)$ & 103 & $61(59)$ & $42(41)$ & \\
\hline \multirow{2}{*}{$\begin{array}{l}\text { History of } \\
\text { family }(\%)\end{array}$} & $(+)$ & 43 & $22(51)$ & $21(49)$ & \multirow{2}{*}{0.319} \\
\hline & $(-)$ & 64 & $40(62)$ & $24(38)$ & \\
\hline \multirow[t]{2}{*}{ Hyperlipidemia(\%) } & $(+)$ & 20 & $12(60)$ & $8(40)$ & \multirow{2}{*}{1.000} \\
\hline & $(-)$ & 87 & $49(56)$ & $37(44)$ & \\
\hline \multirow{2}{*}{ Cigarette(\%) } & $(+)$ & 21 & $10(47)$ & $11(53)$ & \multirow{2}{*}{0.333} \\
\hline & $(-)$ & 86 & $52(60)$ & $34(40)$ & \\
\hline \multirow{2}{*}{ Presyncope(\%) } & $(+)$ & 33 & $15(45)$ & $18(55)$ & \multirow{2}{*}{0.137} \\
\hline & $(-)$ & 74 & $47(63)$ & $27(37)$ & \\
\hline \multirow{2}{*}{ Syncope(\%) } & $(+)$ & 12 & $5(42)$ & $7(58)$ & \multirow{2}{*}{0.353} \\
\hline & $(-)$ & 95 & $57(60)$ & $38(40)$ & \\
\hline \multirow{3}{*}{ NYHA (\%) class } & I & $42(39)$ & $31(74)$ & $11(26)$ & \multirow{3}{*}{0.002} \\
\hline & II & $43(41)$ & $25(58)$ & $18(42)$ & \\
\hline & III & $22(20)$ & $5(23)$ & $17(77)$ & \\
\hline \multirow{2}{*}{ Beta blockers(\%) } & $(+)$ & 100 & $44(44)$ & $56(56)$ & \multirow{2}{*}{0.238} \\
\hline & $(-)$ & 7 & $6(86)$ & $1(14)$ & \\
\hline \multirow{2}{*}{ Amiodarone(\%) } & $(+)$ & 4 & $1(25)$ & $3(75)$ & \multirow{2}{*}{0.309} \\
\hline & $(-)$ & 103 & $61(59)$ & $42(41)$ & \\
\hline \multirow[t]{2}{*}{ Dysopyramide(\%) } & $(+)$ & 8 & $3(37)$ & $5(63)$ & \multirow{2}{*}{0.280} \\
\hline & $(-)$ & 99 & $59(57)$ & $40(43)$ & \\
\hline \multirow{2}{*}{$\begin{array}{l}\text { Calcium channel } \\
\text { blocker(\%) }\end{array}$} & $(+)$ & 4 & $3(75)$ & $1(25)$ & 0635 \\
\hline & $(-)$ & 103 & $59(57)$ & $44(43)$ & 0.035 \\
\hline $\begin{array}{l}\text { The HCM Risk } \\
\text { SCD }\end{array}$ & & $5.7 \pm 4.1$ & $3.6 \pm 1.8$ & $8.7 \pm 4.8$ & $<0.001$ \\
\hline The HCM Risk & $(+)$ & 36 & $5(14)$ & $31(86)$ & $<0.001$ \\
\hline $\operatorname{SCD}(>6 \%)$ & $(-)$ & 71 & $51(82)$ & $14(18)$ & \\
\hline Galectin-3(ng/ml) & & $\begin{array}{l}5.798 \pm 2.52 \\
6\end{array}$ & $4.035 \pm 1.464$ & $8.226 \pm 1.412$ & $<0.001$ \\
\hline LAAPD(mm) & & $42.2 \pm 4.3$ & $41.8 \pm 4.4$ & $42.8 \pm 4.2$ & 0.226 \\
\hline LAV(ml) & & $53.4 \pm 15.1$ & $49.4 \pm 13.8$ & $59.7 \pm 16.5$ & 0.001 \\
\hline $\mathrm{LAVI}\left(\mathrm{ml} / \mathrm{m}^{2}\right)$ & & $29.8 \pm 8.5$ & $27.7 \pm 7.5$ & $33.2 \pm 9.8$ & 0.002 \\
\hline LV EF(\%) & & $66.9 \pm 5.9$ & $67.2 \pm 5.2$ & $66.5 \pm 6.9$ & 0.527 \\
\hline
\end{tabular}


The Importance of Galectin 3 for Risk Stratification and Prognosis in Hypertrophic Cardiomyopathy

\begin{tabular}{|c|c|c|c|c|}
\hline $\operatorname{IVST}(\mathbf{m m})$ & $21.9 \pm 3.9$ & $20.7 \pm 3.8$ & $24.0 \pm 4.5$ & $<U_{\text {.UVI }}$ \\
\hline LVPWT(mm) & $6.4 \pm 3.1$ & $12.9 \pm 3.3$ & $12.8 \pm 2.9$ & 0.854 \\
\hline LVM(g) & $333.2 \pm 85.5$ & $316.7 \pm 92.0$ & $359.9 \pm 76.1$ & 0.012 \\
\hline $\operatorname{LVMI}\left(\mathrm{g} / \mathrm{m}^{2}\right)$ & $180.8 \pm 53.1$ & $173.1 \pm 57.6$ & $194.0 \pm 48.4$ & 0.049 \\
\hline RWTI & $0.62 \pm 0.22$ & $0.62 \pm 0.23$ & $0.62 \pm 0.24$ & 0.977 \\
\hline LVOTO(mmHg) & $26.1 \pm 29.8$ & $25.1 \pm 30.1$ & $28.3 \pm 31.5$ & 0.599 \\
\hline Total cholesterol(mg/dl) & $186.4 \pm 34.9$ & $190.9 \pm 38.5$ & $179.5 \pm 29.9$ & 0.104 \\
\hline C-Reactive protein $(\mathrm{mg} / \mathrm{dl})$ & $3.4 \pm 6.2$ & $3.4 \pm 6.3$ & $3.5 \pm 6.1$ & 0.927 \\
\hline White blood cell $\left(\times 10^{9} / \mathrm{L}\right)$ & $7.9 \pm 1.9$ & $8.0 \pm 2.0$ & $7.8 \pm 1.8$ & 0.659 \\
\hline Blood urine nitrogen(mg/dl) & $14 \pm 4.3$ & $14.0 \pm 4.6$ & $14.2 \pm 4.0$ & 0.817 \\
\hline Creatinin(mg/dl) & $0.8 \pm 0.1$ & $0.8 \pm 0.1$ & $0.8 \pm 0.2$ & 0.293 \\
\hline \multirow{2}{*}{$\begin{array}{l}\text { Paroxysmal atrial } \\
\text { fibrillation(\%) }\end{array}$} & 10 & $4(40)$ & $6(60)$ & \multirow[t]{2}{*}{0.316} \\
\hline & 97 & $58(60)$ & $39(40)$ & \\
\hline \multirow{2}{*}{$\begin{array}{l}\text { Ventricular extra } \\
\text { sistole }\end{array}$} & 73 & $37(51)$ & $36(49)$ & \multirow{2}{*}{0.037} \\
\hline & 34 & $25(73)$ & $9(27)$ & \\
\hline \multirow{2}{*}{$\begin{array}{l}\text { Ventricular } \\
\text { tachycardia(\%) }\end{array}$} & 23 & $8(35)$ & $15(65)$ & \multirow{2}{*}{0.017} \\
\hline & 84 & $53(63)$ & $30(37)$ & \\
\hline \multirow{2}{*}{$\begin{array}{l}\text { Cardiopulmonary } \\
\text { rescucitation(\%) }\end{array}$} & 12 & $1(8)$ & $11(92)$ & \multirow[t]{2}{*}{$<0.001$} \\
\hline & 95 & $61(64)$ & $34(36)$ & \\
\hline \multirow{2}{*}{$\begin{array}{l}\text { ICD } \\
\text { implantation(\%) }\end{array}$} & 11 & $1(9)$ & $10(91)$ & \multirow{2}{*}{0.010} \\
\hline & 96 & $61(63)$ & $35(37)$ & \\
\hline \multirow[t]{2}{*}{ Shock(\%) } & 8 & $1(12)$ & $7(88)$ & \multirow{2}{*}{0.002} \\
\hline & 3 & 0 & $3(100)$ & \\
\hline \multirow{2}{*}{$\begin{array}{l}\text { Admitted with } \\
\text { heart failure(\%) }\end{array}$} & 31 & $12(39)$ & $19(61)$ & \multirow{2}{*}{0.016} \\
\hline & 76 & $50(66)$ & $27(34)$ & \\
\hline
\end{tabular}

Values are the mean \pm standard deviation or number (\%). EF: ejection fraction, HCM: hypertrophic cardiomyopathy, HCM Risk-SCD: predicted 5-year risk of sudden cardiac death in HCM patients, ICD: implantable cardioverter defibrillator, IVST: interventricular septum thickness, LAAPD: left atrium anterior-posterior dimension, LAV. left atrium volume, LAVI: left atrium volume index, LVEDD: left ventricular enddiastolic dimension, LVESD: left ventricular end-systolic dimension, LVM: left ventricular mass, LVMI: left ventricular mass index, LVPWT: left ventricular posterior wall thickness, LVOTO: left ventricular outflow tract obstruction, NYHA: New York Heart Association, RWTI: relative wall thickness index, SCD: sudden cardiac death.

\section{Correlation results of Galectin-3 and other parameters}

Results for correlation between galectin-3 levels, and other parameters in patients are summarized in Table 2 . A statistically significant correlation was observed between galectin-3 and NYHA class $(\%)(0.351, \mathrm{p}<0.001)$, LAV $(\mathrm{ml})(\mathrm{r}=$ $0.434, \mathrm{p}<0.001)$, LAVI $(\mathrm{ml} / \mathrm{m} 2)(\mathrm{r}=0.439,<0.001)$, IVST $(\mathrm{mm})(\mathrm{r}=0.373, \mathrm{p}$ $<0.001)$, LVM (g) $(\mathrm{r}=0.326, \mathrm{p}=0.001)$, LVMI $(\mathrm{g} / \mathrm{m} 2)(\mathrm{r}=0.339, \mathrm{p}=0.001)$, paroxysmal atrial fibrillation (PAF) $(\%)(\mathrm{r}=0.211, \mathrm{p}=0.026)$, ventricular extra systole $(r=0.338, p<0.001)$ ventricular tachycardia $(\%)(r=0.229, p=0.020)$, 
cardiopulmonary resuscitation (CPR) $(\%)(\mathrm{r}=0.319, \mathrm{p}=0.001)$, implantable cardioverter defibrillator (ICD) implantation $(\%)(\mathrm{r}=0.264, \mathrm{p}=0.007)$, admitted heart failure $(\%)(\mathrm{r}=0.275, \mathrm{p}=0.007)$, appropriate shock $(\%)(\mathrm{r}=0.279, \mathrm{p}=$ $0.005)$, the HCM Risk SCD $(r=0.629, p=<0.001)$, the HCM Risk SCD $(>6 \%)$ $(\mathrm{r}=0.617, \mathrm{p}<0.001)$.

Table 2. Correlations coefficients of the relationship between Galectin-3 and other parameters.

\begin{tabular}{|c|c|c|}
\hline Variable & Galect & \\
\hline & $\mathrm{r}$ & $\mathrm{P}$ \\
\hline Age(years) & -0.094 & 0.337 \\
\hline Gender & 0.084 & 0.387 \\
\hline BMI $\left(\mathrm{kg} / \mathbf{m}^{2}\right)$ & 0.064 & 0.511 \\
\hline History of family $(\%)$ & 0.179 & 0.066 \\
\hline Presyncope(\%) & 0.150 & 0.124 \\
\hline Syncope $(\%)$ & 0.126 & 0.196 \\
\hline NYHA (\%) class I & 0.351 & $<0.001$ \\
\hline II & & \\
\hline III & & \\
\hline LAAPD(mm) & 0.179 & 0.066 \\
\hline $\mathbf{L A V}(\mathbf{m l})$ & 0.434 & $<0.001$ \\
\hline $\mathbf{L A V I}\left(\mathbf{m l} / \mathbf{m}^{2}\right)$ & 0.439 & $<0.001$ \\
\hline LV EF $(\%)$ & -0.073 & 0.457 \\
\hline IVST(mm) & 0.373 & $<0.001$ \\
\hline LVPWT(mm) & 0.039 & 0.694 \\
\hline LVM $(g)$ & 0.326 & 0.001 \\
\hline $\operatorname{LVMI}\left(\mathbf{g} / \mathbf{m}^{2}\right)$ & $\mathbf{0 . 3 3 9}$ & 0.001 \\
\hline RWTI & 0.023 & 0.817 \\
\hline LVOTO(mmHg) & 0.048 & 0.629 \\
\hline C-Reactive protein (mg/dl) & 0.084 & 0.393 \\
\hline White blood cell $\left(\times 10^{9} / \mathrm{L}\right)$ & 0.040 & 0.686 \\
\hline Creatinin(mg/dl) & 0.144 & 0.144 \\
\hline PAF(\%) & 0.211 & 0.026 \\
\hline Ventricular extra sistole & 0.338 & 0.001 \\
\hline Ventricular tachycardia (\%) & 0.229 & 0.020 \\
\hline CPR(\%) & 0.319 & 0.001 \\
\hline ICD implantation(\%) & 0.264 & 0.007 \\
\hline Admitted heart failure & 0.275 & 0.003 \\
\hline Apropriate shock & 0.279 & 0.005 \\
\hline The HCM Risk SCD & 0.629 & $<0.001$ \\
\hline The HCM Risk SCD $(>6 \%)$ & 0.617 & $<0.001$ \\
\hline
\end{tabular}

Values are the mean \pm standard deviation or number (\%). CPR: cardiopulmonary resuscitation, EF: ejection fraction, HCM: hypertrophic cardiomyopathy, HCM Risk- SCD: predicted 5-year risk of sudden cardiac death in HCM patients, HF: heart failure, ICD: implantable cardioverter defibrillator, IVST: interventricular septum thickness, LVPWT: left ventricular posterior wall thickness, LAAPD: left atrium anterior-posterior dimension, LAV: left atrium volume, LAVI: left atrium volume index, LVM: left ventricular mass, LVMI: left ventricular mass index, LVOTO: left ventricular outflow tract obstruction, NYHA: New York Heart Association, PAF: paroxysmal atrial fibrillation, RWTI: relative wall thickness index, SCD: sudden cardiac death. 


\section{Univariate and multivariate analysis}

Logistic regression analyses for independent predictors of high-risk predicted five years risk sudden cardiac death have been shown in Table 3. Both in the univariate and multivariate analysis, galectin-3 determined that the HCM Risk SCD is an independent predictor of high-risk (Galectin-3 univariate analysis: odds ratio: 0.999 , confidence interval: $0.998-1.000, \mathrm{p}$ value: $<0.001$; multivariate analysis: odds ratio: 0.999 , confidence interval: $0.998-1.000$, p value: $<0.001)$. In a receiver operating characteristic (ROC) curve analysis, a Galectin-3>6.324 ng/ $\mathrm{ml}$ was identified as an effective cut-off point in the HCM Risk SCD for HCM (area under curve $=0.878,95 \% \mathrm{CI}=0.815-0.942, \mathrm{p}=<0.001$ ). A galectin-3 value of more than $6.324 \mathrm{ng} / \mathrm{ml}$ yielded a with sensitivity, specificity, positive predictive value and negative predictive value of, 83\%, 82\%, 73\%, 93\% (Figure 2).

\section{ROC Curve}

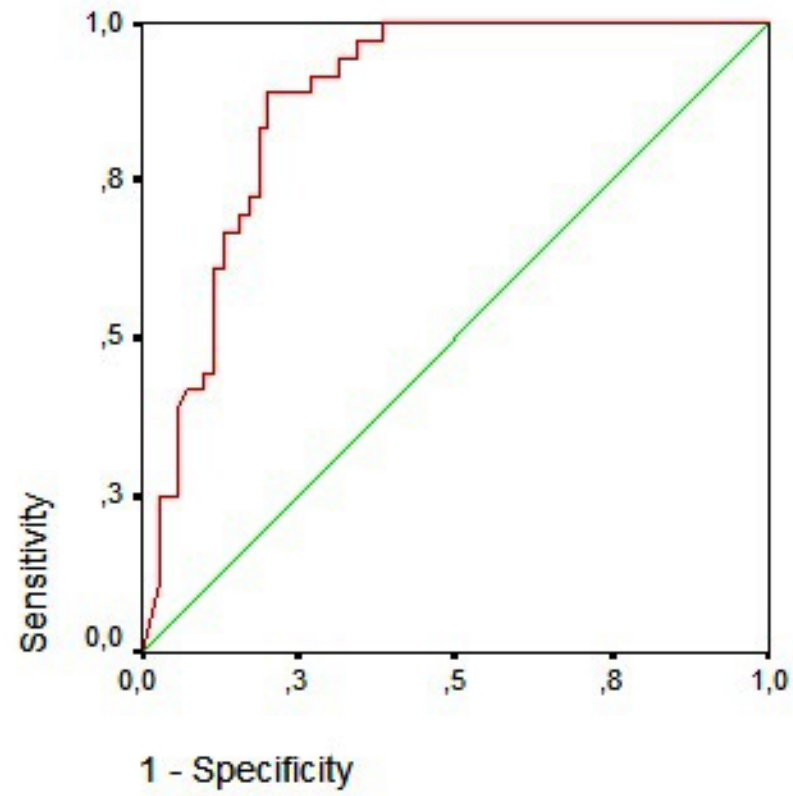

Figure 2. In a receiver operating characteristic (ROC) curve analysis, a Galectin-3>6.324 ng/ml was identified as an effective cut-off point in the HCM Risk SCD for HCM (area under curve = $0.878,95 \% C I=0.815-0.942, p=<0.001)$. 
Table 3. Univariate and multivariate analyses for independent high-risk predictors the HCM Risk SCD.

\begin{tabular}{|c|c|c|c|c|c|c|}
\hline & \multicolumn{2}{|c|}{ Univariate } & \multicolumn{4}{|c|}{ Multivariate } \\
\hline & OR & $\mathrm{CI}$ & $\mathrm{p}$ & OR & $\mathrm{CI}$ & $\mathrm{p}$ \\
\hline Galectin-3 & 0.999 & 0.998-1.000 & $<0.001$ & 0.999 & $\begin{array}{l}0.998- \\
1.000\end{array}$ & $<0.001$ \\
\hline New york heart association & 0.196 & $0.098-0.394$ & $<0.001$ & & & \\
\hline Presyncope & 4.130 & $1.793-9.513$ & 0.001 & & & \\
\hline Admitted with heart failure & 6.778 & $\begin{array}{l}2.771- \\
16.580\end{array}$ & $<0.001$ & & & \\
\hline Paroxysmal atrial fibrillation & 4.242 & $\begin{array}{l}1.192- \\
15.100\end{array}$ & 0.026 & & & \\
\hline Ventricular tachycardia & 9.788 & $\begin{array}{l}3.473- \\
27.585\end{array}$ & $<0.001$ & & & \\
\hline Left atrium volume & 0.961 & $0.936-0.988$ & 0.004 & & & \\
\hline Left atrium volume index & 0.937 & $0.895-0.982$ & 0.007 & & & \\
\hline $\begin{array}{l}\text { Interventricular septum } \\
\text { thickness }\end{array}$ & 0.846 & $0.768-0.932$ & 0.001 & & & \\
\hline Left ventricular mass & 0.995 & $0.990-0.999$ & 0.027 & & & \\
\hline
\end{tabular}

CI: confidence interval, HCM: hypertrophic cardiomyopathy, OR: odds ratio, SCD: sudden cardiac death

\section{Discussion}

In this study, we investigated whether galectin-3 levels could be a potential predictor of cardiac events, focusing on arrhythmic events, admission to hospital, prognosis and the predicted the HCM Risk-SCD in patients with HCM. One of the results of this study; There was a significant difference between the Galectin-3 level and the 5-year risk of sudden cardiac death, moreover galactin-3 level was significantly higher in patients with the HCM Risk-SCD above $6 \%$.

Galectin-3, which is secreted by macrophages, has been known for its significant role in mediating cardiac fibrosis and inflammation. Serum levels of galectin-3 increases when the destruction and remodeling in the tissues is excessive. Moreover, this increase in galectin-3 levels were shown to be associated with 
poor prognosis in recent studies. Galectin-3 was shown to promote cardiac fibrogenesis by activating resting fibroblast $(8,9)$. Supporting this information had been shown significantly correlation between galectin-3 levels and LGE MRI verified replacement fibrosis in nonischemic dilate cardiomyopathy.

The cause of this abnormal fibrosis is related secondary myocardial ischemia caused by to chaotic, disorganized myocytes and abnormal mass of ventricle. In HCM patients there is an imbalance of supply and demand mismatches in coronary blood flow of myocardium, which is due to abnormally increased mass and abnormal intramural coronary arteries with thickened walls and narrowed lumen $(10,11)$. Increased stress and injury cause myocardial damage. This situation causes immune cells to accumulate into the myocardium, resulting in activation of resident fibroblast and deposition of procollagen into extracellular matrix which leads to cardiac fibrosis. As a result of all these negative mechanisms can contribute to myocardial remodeling $(12,13)$. In this study, there are significant correlations between plasma Galectin-3 levels and LVM, LVMI, interventricular septum thickness.

In HCM patients the disorganized cellular architecture and replacement fibrosis predispose to serve an electrically unstable substrate and nidus for reentry ventricular tachyarrhythmias and sudden death (13). In this study, there were positive correlations between serum Galectin-3 levels and percentage of ventricular extrasystole, ventricular tachycardia in HCM group. Furthermore, requirement for CPR and ICD implantation were positive correlated with serum Galectin-3 levels.

Several studies already proved galectin-3 could be a well prognostic marker in Heart failure (HF) for predicting cardiovascular mortality and re-hospitalization after multivariate analysis but not only patient with low ejection fraction (14). A previous study had demonstrated that galectin-3 levels were significantly elevated in patients with HF with preserved ejection fraction $(14,16)$. Galectin-3 might provide an early warning marker for patients who are at risk for development of HF and may allow medical treatment. In this study NYHA class III symptom defining patients were significantly higher in higher galectin-3 levels. Galectin 3 levels and percentage of hospitalization due to heart failure were significantly correlated.

Although the main causes of cardiac mortality in HCM are SCD, heart failure or thromboembolic events, SCD has always been the most frightening complication ever since it was first described which actually makes a lot of sense. Although there are many new parameters for risk assessment today, very few of them are accepted for clinical use the HCM Risk SCD assessment is one of them which 
entered the ESC guideline in 2014. In a recent study evaluating patients with HCM, there was no correlation between galectin levels and the HCM Risk SCD (17). This may be due to the fact that the number of patients was relatively small, or because of the short duration of follow-up, symptomatic changes could not be followed for as long as enough time. As is known, the structural changes in HCM occasionally may be delayed until later in midlife $(18,19)$. But, in that study, the mean age of patients included in the study were 20 years old. Unlike that study, in our study, there was a significant correlation between galectin-3 levels and the HCM Risk SCD. Moreover, in galectin-3 was observed as an independent highrisk predictor for the HCM Risk SCD in univariate and multivariate analyses.

\section{Study limitations}

There are several limitations associated with the study. Study population was relatively small and the other oxidative stress markers were not assessed. Pediatric patients were not included in our study. The follow-up period of the study was not long enough for monitoring patients and it is an important disadvantage.

\section{Conclusion}

These results shape the concept of considering galectin-3 as a new target for therapeutic intervention or recognizing patients with high risk for sudden cardiac death or malign arrythmias at an early stage of compensated hypertrophy in failure-prone hearts. Our results suggest that galectin-3 may provide some information about disease severity and galectin-3 is an independent predictor of high risk for HCM Risk-SCD in HCM. Therefore, an early recognition of failureprone hearts and intervention with new anti-inflammatory and antifibrotic agents might provide additional benefit over existing treatment strategies.

\section{References}

1. ElliottPM,Anastasakis A, BorgerMA, Borggrefe M,CecchiF, CharronP, et al. 2014 ESC Guidelines on diagnosis and management of hypertrophic cardiomyopathy: the Task Force for the Diagnosis and Management of Hypertrophic Cardiomyopathy of the European Society of Cardiology (ESC). Eur Heart J 2014;35:2733-79.

2. Begley DA, Mohiddin SA, Tripodi D, Winkler JB, Fananapazir L. Efficacy of implantable cardioverter defibrillator therapy for primary and secondary prevention of sudden cardiac death in hypertrophic cardiomyopathy. Pacing Clin Electrophysiol 2003;26:1887-96.

3. Teare D. Asymmetrical hypertrophy of the heart in young adults. $\mathrm{Br}$ Heart J 1958;20:1-8 
4. O’Mahony C, Jichi F, Pavlou M, Monserrat L, Anastasakis A, Rapezzi $\mathrm{C}$, et al. Hypertrophic Cardiomyopathy Outcomes Investigators. A novel clinical risk prediction model for sudden cardiac death in hypertrophic cardiomyopathy(HCM Risk-SCD). Eur Heart J 2014;35:2010-20.

5. Ho JE, Liu C, Lyass A, Courchesne P, Pencina MJ, Vasan RS, et al. Galectin-3, a marker of cardiac fibrosis, predicts incident heart failure in the community. J Am Coll Cardiol 2012;60(14):1249-56.

6. Schiller NB, Shah PM, Crawford M, DeMaria A, Devereux R, Feigenbaum $\mathrm{H}$, et al. Recommendations for quantitation of the left ventricle by two dimensional echocardiography. American Society of Echocardiography Committee on Standards, Subcommittee on Quantitation of TwoDimensional Echocardiograms. J Am Soc Echocardiogr 1989;2:358-67.

7. Devereux RB, Koren MJ, de Simone G, Okin PM, Kligfield P. Methods for detection of left ventricular hypertrophy: application to hypertensive heart disease. Eur Heart J 1993;14:8-15.

8. Vergaro G, Del Franco A, Giannoni A, Prontera C, Ripoli A, Barison A, et al. Galectin3 and myocardial fibrosis in nonischemic dilated cardiomyopathy. Int J Cardiol 2015;184:96-100.

9. Yu L, Ruifrok WP, Meissner M, Bos EM, van Goor H, Sanjabi B, et al. Genetic and pharmacological Inhibition of Galectin-3 Prevents Cardiac Remodeling by Interfering with Myocardial Fibrogenesis. Circ Heart Fail 2013;6:107-17.

10. Nienaber CA, Gambhir SS, Mody FV, Ratib O, Huang SC, Phelps $\mathrm{ME}$, et al. Regional myocardial blood flow and glucose utilization in symptomatic patients with hypertrophic cardiomyopathy. Circulation 1993;87:1580-90.

11. Amin HZ, Amin LZ, Wijaya IP. Galectin-3: a novel biomarker for the prognosis of heart failure. Clujul Med. 2017;90(2):129-32.

12. Nienaber CA, Gambhir SS, Mody FV, Ratib O, Huang SC, Phelps $\mathrm{ME}$, et al. Regional myocardial blood flow and glucose utilization in symptomatic patients with hypertrophic cardiomyopathy. Circulation 1993;87:1580-90.

13. Özyılmaz S, Püşüroğlu H. Assessment of the relationship between the ambulatory electrocardiography-based micro T-wave alternans and the predicted risk score of sudden cardiac death at 5 years in patients with hypertrophic cardiomyopathy. Anatol J Cardiol 2018;20(3):165-73. 
14. Zile MR, DeSantis SM, Baicu CF, Prescott CB, Stroud RE, Thompson SB, el al. Plasma Galectin-3 Levels in Patients with Structural and Clinical Manifestations of Hypertensive Heart Disease: Relationship to Determinants of Matrix Composition. Abstract 12433. Circulation. 2010;122:A12433.

15. Maron MS, Zenovich AG, Casey SA, Link MS, Udelson JE, Dorothee M et al. Significance and relationship between magnitude of left ventricular hypertrohy and heart failure symptoms in hypertrophic cardiomyopathy. Am J Cardiol 2005 Jun 1;95(11):1329-33.

16. Besler C, David Lang D, Daniel Urban D, Rommel KP, Von Roeder M, Fengler K,et al.Plasma and Cardiac Galectin-3 in Patients With Heart Failure Reflects Both Inflammation and Fibrosis Implications for Its Use as a Biomarker. Circ Heart Fail 2017 Mar;10(3):e003804.

17. Gawor M, Śpiewak M, Janas J, Kożuch K, Wróbel A, Mazurkiewicz Ł, el al. The usefulness of soluble ST2 and galectin-3 as novel biomarkers for better risk stratification in hypertrophic cardiomyopathy. Kardiol Pol 2017;75(10):997-1004.

18. Kampmann C, WiethoffCM, Wenzel A, Stolz G, Betancor M, Wippermann $\mathrm{CF}$, et al. Normal values of $\mathrm{M}$ mode echocardiographic measurements of more than 2000 healthy infants and children in central Europe. Heart 2000;83:667-672.

19. Maron BJ, Rowin EJ, Casey SA, Lesser JR, Garberich RF, McGriff DM, et al. Hypertrophic cardiomyopathy in children, adolescents, and young adults associated with low cardiovascular mortality with contemporary management strategies. Circulation 2016;133(1):62-73. 